\title{
RESEARCH
}

\section{Prolactin-releasing peptide improved leptin hypothalamic signaling in obese mice}

\author{
Martina Holubová1, Lucie Hrubá1, Barbora Neprašová1,2, Zuzana Majerčíková1, Zdeňka Lacinová3,4, \\ Jaroslav Kuneš1,2, Lenka Maletínská1 and Blanka Železná1 \\ IInstitute of Organic Chemistry and Biochemistry, The Czech Academy of Sciences, Czech Republic \\ IInstitute of Physiology, The Czech Academy of Sciences, Prague, Czech Republic \\ ${ }^{3}$ Institute for Clinical and Experimental Medicine, Prague, Czech Republic \\ 4First Faculty of Medicine, Charles University in Prague and General University Hospital, Prague, Czech Republic \\ Correspondence should be addressed to L Maletínská: maletin@uochb.cas.cz
}

\begin{abstract}
The situation following anti-obesity drug termination is rarely investigated, eventhough a decrease in body weight needs to be sustained. Therefore, this study examined the impact of twice-daily peripheral administration of $5 \mathrm{mg} / \mathrm{kg}$ [N-palm- $\gamma \mathrm{Glu}$-Lys $\left.{ }^{11}\right]$ prolactin-releasing peptide 31 (palm ${ }^{11}$-PrRP31) in mice with diet-induced obesity (DIO from consuming a high-fat diet) after 28 days of treatment (palm ${ }^{11}$-PrRP31 group) and after 14 days of peptide treatment followed by 14 days of discontinuation (palm ${ }^{11}$ PrRP31 + saline group). At the end of the treatment, cumulative food intake, body weight and subcutaneous fat weight/body weight ratio and leptin plasma level were reduced significantly in both the palm ${ }^{11-P r R P 31}$ group and the palm ${ }^{11}$-PrRP31+saline group compared to the saline control group. This reduction correlated with significantly increased FOSB, a marker of long-term neuronal potentiation, in the nucleus arcuatus and nucleus tractus solitarii, areas known to be affected by the anorexigenic effect of palm ${ }^{11-P r R P 31}$. Moreover, activation of leptin-related hypothalamic signaling was registered through an increase in phosphoinositide-3-kinase, increased phosphorylation of protein kinase $B(P K B, A K T)$ and enhanced extracellular signal-regulated kinase 1/2 phosphorylation. Besides, lowered apoptotic markers c-JUN N-terminal kinase and c-JUN phosphorylation were registered in the hypothalami of both palm ${ }^{11-P r R P 31-t r e a t e d}$ groups. This study demonstrates that palm ${ }^{11-P r R P 31}$ positively affects feeding and leptinrelated hypothalamic signaling, not only after 28 days of treatment but even 14 days after the termination of a 14-day long treatment without the yo-yo effect.
\end{abstract}

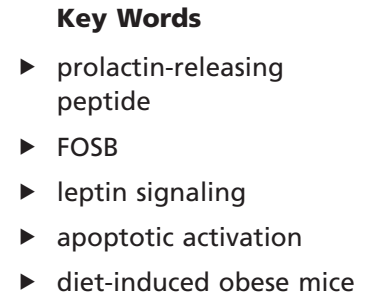

Journal of Molecular Endocrinology (2018) 60, 85-94

\section{Introduction}

Obesity is a common metabolic disease, but its effective noninvasive therapy remains scarce (Bray et al. 2016). Several anti-obesity drugs - analogs of neurotransmitters decreasing appetite or craving for food - were withdrawn from the market because of their severe psychiatric or cardiovascular side effects (for reviews, see
Rodgers et al. 2012, Bray \& Ryan 2014, Manning et al. 2014). Conversely, analogs of anorexigenic peptides promise minimal side effects during long-term antiobesity treatment (Arch 2015, Patel 2015, Bray et al. 2016). Liraglutide, a peptide analog of glucagon-like peptide-1 (GLP1) used to treat type 2 diabetes, has been 
recently approved for obesity treatment in the USA (Saxenda). A selective peptide agonist of receptor MC4R, a receptor of anorexigenic neuropeptide $\alpha$-melanocytestimulating hormone $(\alpha-\mathrm{MSH})$, was shown to attenuate body weight in both rats and pigs with DIO (diet-induced obesity) (Fosgerau et al. 2014).

Both liraglutide and the $\alpha$-MSH analog mentioned have palmitic acid attached in order to improve the pharmacokinetics of the drugs. Similarly, palmitoylation of anorexigenic neuropeptide prolactin-releasing peptide (PrRP) increased the stability and half-life of the peptide. In addition, unlike natural PrRP, palmitoylated PrRP accomplished a central anorexigenic effect both in mice and rats with DIO after peripheral administration (Maletínská et al. 2015, Holubova et al. 2016). Likewise, myristoylated and palmitoylated PrRP31 analogs with C-terminal Phe ${ }^{31}$ modified with non-coded amino acids showed in vitro biological activity and an acute effect on food intake after peripheral administration similar to natural PrRP31 (Prazienkova et al. 2016). Similar biological properties were registered also with [N-palm- $\gamma$ Glu-Lys ${ }^{11}$ ] PrRP31 (palm ${ }^{11}$ PrRP31) with palmitoyl positioned in the middle of the peptide chain (Pražienková et al. 2017).

Central effect of PrRP was demonstrated after its intracerebroventricular administration that inhibited food intake and body weight gain and induced c-FOS in brain areas, such as PVN, regulating food intake (Lawrence et al. 2002). In PVN, and also in periventricular nucleus and dorsomedial nucleus (DMN) of the hypothalamus, reticular nucleus of the thalamus, nucleus tractus solitarii (NTS) and area postrema PrRP receptor, GPR10 is expressed (Roland et al. 1999).

PrRP neurons in the brainstem and hypothalamus were reported to express leptin receptors (Ellacott et al. 2002). In DMH PrRP neurons, leptin activated signal transducer and activator of transcription protein 3 (STAT3) (Takayanagi et al. 2008) while anorexigenic effect of leptin was impaired in PrRP- or GPR10-knockout mice (Dodd \& Luckman 2013). It connected leptin signaling to the anorexigenic effect of PrRP, but the mechanism has not been explained yet.

After termination of long-term administration of an anti-obesity drug, it is necessary to sustain the decrease in body weight in order to minimize the yo-yo effect. However, the situation after anti-obesity drug termination is rarely investigated thoroughly. Therefore, in this study, food intake, body and organ weight and biochemical and hormonal parameters were examined in mice with DIO after a 28-day treatment with palm ${ }^{11}$-PrRP31 and 28 days after the beginning of a 14-day treatment followed by
14 days without peptide treatment. In addition, the impact of such discontinued treatment was studied in the hypothalamic nuclei and nucleus tractus solitarii that were activated by lipidized PrRP in our previous studies (Maletínská et al. 2015, Pražienková et al. 2017). In addition, the effect of cessation of the treatment on hypothalamic signaling related to food intake regulation was determined.

\section{Materials and Methods}

\section{Synthesis of palm ${ }^{11-P r R P 31}$}

Human palmitoylated PrRP31 (SRTHRHSMEI K (N- $\gamma$-E (N-palm)) TPDINPAWYASRGIRPVGRF-NH $\left.{ }_{2}\right)$, palm ${ }^{11}$ PrRP31, was synthesized and purified as described previously (Maletínská et al. 2015). Palmitoylation in position 11 of the PrRP31 analog was performed on a fully protected peptide on resin as the last step (Pražienková et al. 2017). The purity and identity of the peptide was determined by analytical high-performance liquid chromatography and using a Q-TOF micro MS technique (Waters, Milford, MA, USA).

\section{Study in DIO mice administered with palm"1-PrRP31 for $\mathbf{2 8}$ or $\mathbf{1 4}$ days and tissue dissection}

All of the animal experiments followed the ethical guidelines for animal experiments and the Act of the Czech Republic Nr. 246/1992 and were approved by the Committee for Experiments with Laboratory Animals of the Academy of Sciences Czech Republic.

Male C57BL/6 mice from Charles Rivers Laboratories were housed at a temperature of $23^{\circ} \mathrm{C}$ with a daily 12 -h light/darkness cycle (lights on at 06:00 h). The mice were given ad libitum water and a standard rodent chow diet (Ssniff Spezialdiäten GmbH, Soest, Germany). Beginning at 8 weeks of age, mice were fed with a high-fat (HF) diet for 12 weeks to induce obesity. The energy content of the HF diet was $5.3 \mathrm{kcal} / \mathrm{g}$, with 13,60 and $27 \%$ of the calories derived from protein, fat and carbohydrate, respectively (Maletínská et al. 2015). Food intake and body weight were monitored weekly. Mice resistant to the HF diet were withdrawn from the experiment (approximately 10\% of the original number). At the age of 19 weeks, the mice were divided into three groups ( $n=14$ animals) and placed into separate cages with free access to HF diet and water.

Mice were subcutaneously (SC) injected twice a day with saline for 28 days (saline group), with palm ${ }^{11}$-PrRP31 $\left(5 \mathrm{mg} / \mathrm{kg}\right.$ ) for 28 days (palm ${ }^{11}$-PrRP31 group) or with 
palm ${ }^{11}$-PrRP31 $(5 \mathrm{mg} / \mathrm{kg})$ for 14 days and for the following 14 days with saline (palm11-PrRP31+saline group). The last injection was on afternoon of day 28, before the food was removed. On day 29 , at the end of the treatment, overnight-fasted mice were killed by decapitation $(n=10)$. The trunk blood was collected, and plasma was separated and stored at $-20^{\circ} \mathrm{C}$. The intraperitoneal adipose tissue (IPAT), the subcutaneous adipose tissue (SCAT), the brown adipose tissue (BAT) and the liver were dissected, weighed and stored at $-70^{\circ} \mathrm{C}$. The brains were removed, and the hypothalami were dissected and lysed in lysis buffer (Špolcová et al. 2015). The remaining four mice from each group were used for immunohistochemistry.

\section{Determination of hormonal and biochemical parameters in blood and plasma}

Glucose levels were measured in whole blood using a Glucocard glucometer (Arkray, Kyoto, Japan). In the plasma, insulin concentrations were measured with radioimmunoassay (RIA) and leptin concentrations were measured with enzyme-linked immunoassay (ELISA) (Millipore). The plasma triglyceride levels were measured by quantitative enzymatic reaction (Sigma), and the free fatty acids (FFA) levels were determined using a colorimetric assay (Roche). Cholesterol was determined by colorimetric assay (Erba Lachema, Brno, Czech Republic). All measurements were performed according to the manufacturer's instructions.

\section{FOSB immunohistochemistry}

After the treatment, 4 mice from each group were deeply anesthetized with sodium pentobarbital $(50 \mathrm{mg} / \mathrm{kg}$, intraperitoneally) and perfused transcardially as previously described (Pirnik et al. 2015). The brains were dissected and cut into coronal sections of $30 \mu \mathrm{m}$ thickness at $-22^{\circ} \mathrm{C}$ using a Cryocut (Leica Microsystems Nussloh $\mathrm{GmbH}, \mathrm{Germany)}$ and processed as described previously (Pirnik et al. 2015) using a monoclonal mouse FOSB protein antibody (1:2000, Abcam 11959). Quantitative assessment of immunostained cells was performed under light microscope (Prima Star, Carl Zeiss), and representative pictures were captured using an Olympus DP 70 digital camera. The FOSB immunoreactive cells were counted separately on each side of the appropriate coronal sections (2-3 sections per animal) within the arcuate nucleus (ARC) (from $-1.58 \mathrm{~mm}$ bregma to bregma $-1.94 \mathrm{~mm}$ ), paraventricular nucleus (PVN) (from bregma
$-0.7 \mathrm{~mm}$ to bregma $-0.94 \mathrm{~mm}$ ), the dorsomedial nucleus (DMN) (from bregma $-1.82 \mathrm{~mm}$ to bregma $-1.94 \mathrm{~mm}$ ) and the nucleus tractus solitarii (NTS) (from bregma $-7.48 \mathrm{~mm}$ to bregma $-7.32 \mathrm{~mm}$ ) according to Franklin and Paxinos' atlas of the mouse brain (Franklin \& Paxinos 1997).

\section{Western blotting}

Hypothalami were processed, and western blotting was performed as previously described (Špolcová et al. 2015).

The following primary antibodies were used for western blotting: insulin receptor substrate 1 (IRS1), phospho-IRS1 (Ser612), phosphoinositide-3-kinase (PI3 kinase), phospho-AKT (Ser473), total Akt, phosphosignal-regulated kinase $1 / 2$ phosphorylation (MAPK/ ERK1/2), total MAPK/ERK1/2, c-JUN, phospho-c-JUN and c-JUN N-terminal kinase (JNK), total STAT3, phosphoSTAT3, SOCS3 (Cell Signaling Technology); leptin receptor (long form OB-R) and phospho-leptin receptor (Tyr1141) (Thermo Fisher Scientific) and beta-actin (Sigma Aldrich). The following secondary antibodies were used: anti-mouse IgG HRP-linked antibody and anti-rabbit IgG HRP-linked antibody (Cell Signaling Technology).

\section{Determination of mRNA expression}

IPAT, SCAT, BAT and liver were processed as described previously (Maletínská et al. 2015). Determination of the mRNA expression of Lep (leptin) in IPAT and SCAT, Ucp1 (uncoupling protein-1) in BAT and Srebf (sterol regulatory element-binding protein-1), Acaca (acetyl CoA carboxylase), Lpl (lipoprotein lipase), Cpt1a and Cpt1b (carnitine palmitoyltransferase $1 \mathrm{a}$ and $1 \mathrm{~b}$ ) in the liver was performed using an ABI PRISM 7500 instrument (Applied Biosystems). The expression of $B 2 m$ (beta-2microglobulin) was used to compensate for variations in input RNA amounts and the efficiency of reverse transcription.

\section{Statistics}

Statistical analysis of the DIO model was performed using unpaired $t$-tests or one-way ANOVA with a Bonferroni's post hoc test as indicated in the figure and table legends. The differences between the control and treated groups were considered to be significant at $P<0.05$. Linear regression analysis was used to test the relationship between leptin plasma levels and particular fat tissues. 
A Cumulative food intake

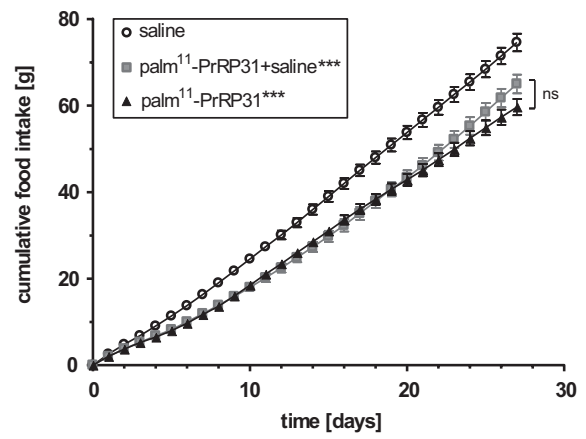

B Body weight

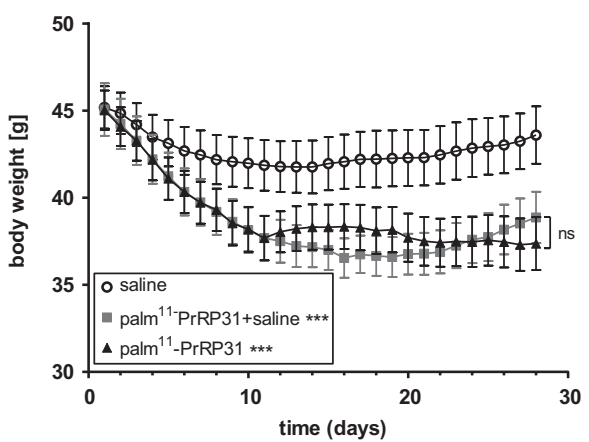

C Body weight change

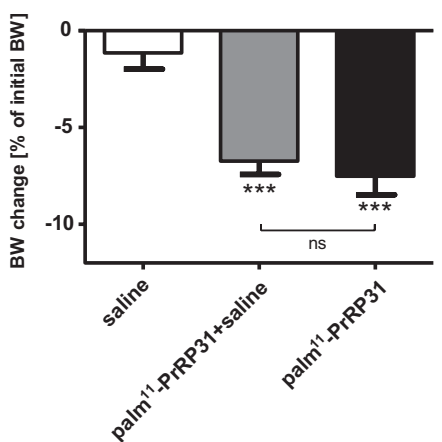

Figure 1

Chronic effect of palm ${ }^{11}$-PrRP31 on (A) food intake and (B and C) body weight in DIO mice. Mice were subcutaneously injected twice a day with saline for 28 days (saline group), with palm ${ }^{11}$-PrRP31 $\left(5 \mathrm{mg} / \mathrm{kg}\right.$ ) for 28 days (palm ${ }^{11}$-PrRP31 group) or with palm ${ }^{11}$-PrRP31 (5 mg/kg) for 14 days and for the following 14 days with saline (palm ${ }^{11}$-PrRP31 + saline group). Data are presented as means \pm S.E.M. Statistical analysis was performed by One-way ANOVA with Bonferroni post hoc test, significance is $* * * P<0.001$ vs the saline treated group $(n=14)$. No significant difference was found between the groups treated with palm ${ }^{11}$-PrRP31 for 14 or 28 days.

\section{Results}

\section{Effect of the treatment on organ weight and biochemical parameters}

In both the palm ${ }^{11}$-PrRP31 group and the palm ${ }^{11}$ PrRP31+saline group, cumulative food intake was lowered similarly and significantly from day 9 to day 28 compared to the saline control group (Fig. 1A). Moreover, at the end of the experiment, body weight and SCAT weight/body weight ratio of the palm¹1-PrRP31 group and the palm ${ }^{11}$-PrRP31+saline group were similar and were significantly lower than those of the saline control

Table 1 Metabolic parameters analyzed in fasted blood plasma and organs weights of DIO mice at the end of experiment.

\begin{tabular}{|c|c|c|c|}
\hline GROUP/parameter & Saline & $\begin{array}{c}\text { Palm11. } \\
\text { PrRP31 + saline }\end{array}$ & Palm11-PrRP31 \\
\hline Glucose (mmol/L) & $8.06 \pm 0.45$ & $8.37 \pm 0.30$ & $8.65 \pm 0.40$ \\
\hline Insulin (ng/mL) & $1.55 \pm 0.30$ & $1.28 \pm 1.16$ & $1.10 \pm 0.17$ \\
\hline Leptin (ng/mL) & $75.86 \pm 7.70$ & $42.40 \pm 7.01 * *$ & $35.29 \pm 7.94 * *$ \\
\hline $\begin{array}{l}\text { Triglycerides } \\
\text { (mg/mL) }\end{array}$ & $0.22 \pm 0.28$ & $0.36 \pm 0.04 *$ & $0.26 \pm 0.06$ \\
\hline FFA (mmol/L) & $0.52 \pm 0.02$ & $0.54 \pm 0.03$ & $0.49 \pm 0.04$ \\
\hline $\begin{array}{l}\text { Cholesterol } \\
(\mathrm{mmol} / \mathrm{L})\end{array}$ & $3.94 \pm 0.22$ & $3.70 \pm 0.21$ & $3.20 \pm 0.25 *$ \\
\hline SCAT/bw (\%) & $7.44 \pm 0.50$ & $5.25 \pm 0.36 * *$ & $4.74 \pm 0.49 * *$ \\
\hline IPAT/bw (\%) & $4.37 \pm 0.31$ & $4.42 \pm 0.20$ & $5.04 \pm 0.29$ \\
\hline Liver/bw (\%) & $3.63 \pm 0.35$ & $3.30 \pm 0.08$ & $3.22 \pm 0.14$ \\
\hline
\end{tabular}

Data are presented as means \pm S.E.M. Statistical analysis was performed by unpaired $t$-test. Significance is $* P<0.05, * * P<0.01, * * * P<0.001$ vs the saline-treated group $(n=10)$. No significant difference was found between the groups treated with palm¹1-PrRP31 for 14 or 28 days.

FFA, free fatty acids; IPAT, intraperitoneal adipose tissue; SCAT, subcutaneous adipose tissue.

(c) 2018 Society for Endocrinology Published by Bioscientifica Ltd. Printed in Great Britain group (Fig. 1B and Table 1). IPAT weight/body weight ratio remained unaffected by both methods of palm ${ }^{11}$-PrRP31 treatment (Table 1).

A decrease in liver weight/body weight ratio was similar in both palm11-PrRP31-treated groups but was not significant compared to the saline group (Table 1). Blood glucose, as well as insulin, triglycerides, FFA and cholesterol, in plasma were not affected significantly by both methods of palm ${ }^{11}$-PrRP31 treatment (Table 1). However, plasma leptin was attenuated significantly in both palm ${ }^{11}$-PrRP31 - and palm ${ }^{11}$-PrRP31 + saline-treated mice compared to saline controls (Table 1).

It was demonstrated that leptin plasma levels correlated positively with subcutaneous adipose tissue $(r=0.852, P<0.001)$ in all three groups studied. This was not due to palm11-PrRP31 treatment because a similar relationship was seen even in control group $(r=0.642$, $P<0.001)$. However, there was no relationship between leptin and IPAT ( $r=0.003$, non-significant).

\section{Activation of neurons in brain areas connected with food intake regulation}

Neuronal activity was followed at the end of the experiment in three hypothalamic nuclei connected with food intake regulation - PVN, ARC, DMN and in the NTS by FOSB immunohistochemistry. Significantly increased FOSB was registered in ARC and NTS but not PVN and DMN in the palm ${ }^{11-P r R P 31}$ group compared to saline controls (Fig. 2). FOSB in the palm11-PrRP31+ saline group was increased significantly only in NTS in comparison with the saline control group. 


\section{A The FOSB immunoreactive cells activation}

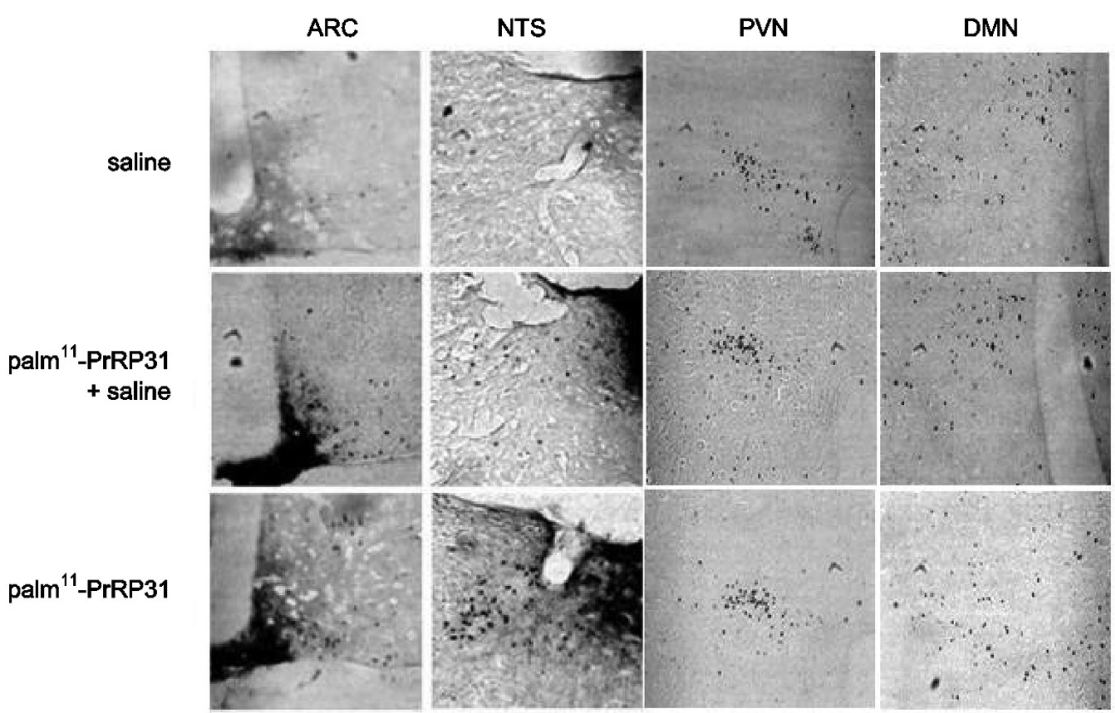

B The amount of immunoreactive cells

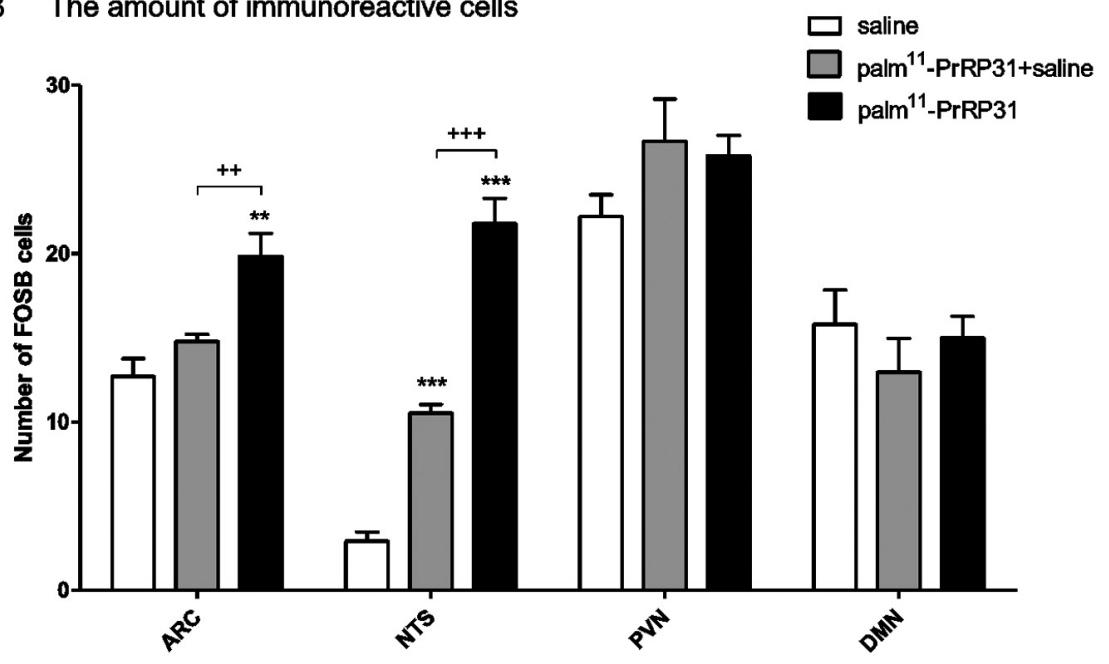

Figure 2

(A) The FOSB immunoreactive cells activation and (B) the amount of immunoreactive cells in selected brain nuclei (ARC, NTS, PVN, DMN) involved in food intake regulation. Mice were subcutaneously injected twice a day with saline for 28 days (saline group), with palm 11 -PrRP31 ( $5 \mathrm{mg} / \mathrm{kg}$ ) for 28 days (palm ${ }^{11}$-PrRP31 group) or with palm ${ }^{11}$-PrRP31 $(5 \mathrm{mg} / \mathrm{kg})$ for 14 days and for the following 14 days with saline (palm ${ }^{11}$ PrRP31 + saline group). After the treatment, mice were deeply anesthetized and perfused transcardially. The brains were dissected and cut into coronal sections. The FOSB immunoreactive cells were counted separately on each side of the appropriate coronal sections. Data are presented as means \pm S.E.M. Statistical analysis was performed by unpaired $t$-test. Significance is $* * P<0.01$, $* * * P<0.001$ vs the saline treated group, ${ }^{++} P<0.01$, ${ }_{+++}^{+}<0.001$ palm ${ }^{11}$-PrRP31 + saline vs palm ${ }^{11}$ PrRP31 ( $n=4)$. ARC, nucleus arcuatus; NTS, nucleus of solitary tract; PVN, paraventricular nucleus; DMN, dorsomedial nucleus.

\section{Impact of the treatment on leptin-related signaling pathways in hypothalamus}

Signaling pathways were measured in hypothalamic lysates using Western blotting in all three experimental groups $(n=8)$. As demonstrated in Fig. 3, the palm ${ }^{11}$ PrRP31 group showed a significant increase in phosphorylation of IRS1 and AKT, and both the palm ${ }^{11}$ PrRP31 and the palm ${ }^{11}$-PrRP31 + saline groups showed PI3 kinase activation. Phosphorylation of MAPK/ERK1/2 was increased in both peptide-treated groups compared to the saline-treated group (Fig. 3).

The apoptotic pathway in the hypothalami of both palm11-PrRP31 and palm11-PrRP31+saline groups was attenuated as shown by decreased levels of JNK and phospho-c-JUN/c-JUN ratio; however, total c-JUN was increased by the treatment (Fig. 4). There was no significant change in leptin STAT3 signaling and inhibition of this signaling through SOCS3 was attenuated in palm ${ }^{11}$ PrRP31-treated compared to saline-treated group (Fig. 4). Significant increase of phosphorylated leptin receptor/ total leptin receptor was observed in palm11-PrRP31treated group and decreased total leptin receptor in the same group compared to saline-treated group (Fig. 5).

\section{Impact of the treatment on mRNA expression of selected genes}

BAT Ucp1 mRNA expression was increased in palm ${ }^{11}$ PrRP31 mice but not palm ${ }^{11-P r R P 31}+$ saline mice compared to saline controls (Fig. 6A). Lep mRNA expression tended toward a non-significant decrease in SCAT (Fig. 6B) and 
Leptin-related signaling pathways in hypothalamus - western blotting

\begin{tabular}{|c|c|c|c|c|}
\hline $\mathrm{kDa}$ & saline & $\begin{array}{c}\text { palm }{ }^{11} \text {-PrRP31 } \\
\text { + saline }\end{array}$ & palm 11-PrRP31 & antibody \\
\hline 180 & E. & w 18 & $=0$ & IRS1 \\
\hline 85 & $-5-m=$ & $-\infty-\infty$ & $\mathrm{eve}$ & PI3 kinase \\
\hline 60 & --- & $-0-0$ & 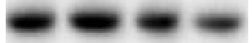 & p-AKT Ser473 \\
\hline 60 & 00 & $\mathbf{0 0 6}$ & $=000$ & AKT \\
\hline 42,44 & $-m-m$ & $-1-0$ & $-0-0$ & p-ERK \\
\hline 42,44 & $-5-5$ & $-5=5$ & $--m-$ & ERK \\
\hline 42 & 0000 & 0000 & 0006 & $\beta$-actin \\
\hline
\end{tabular}

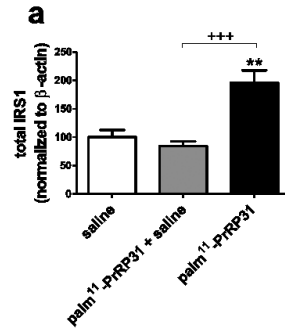

d

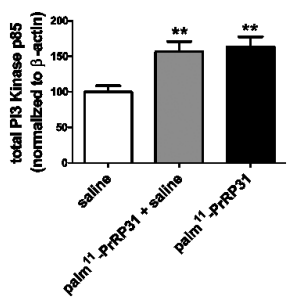

b

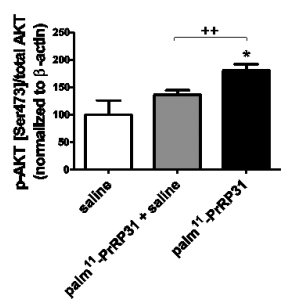

e

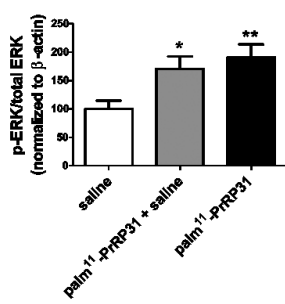

C

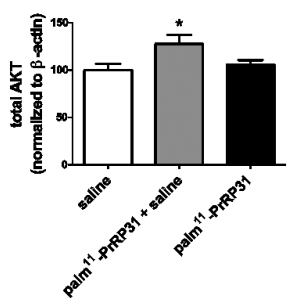

f

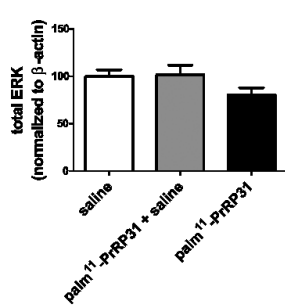

Figure 3

Signaling pathways in hypothalamus. Signaling pathways were measured in hypothalamic lysates using Western blotting. Densitometric quantification of Western blots is normalized to $\beta$-actin. Leptin-related signaling pathways - total IRS1, total PI3 kinase, phospho-AKT (Ser473), total AKT, phospho-ERK1/2 and total ERK. IRS1, insulin receptor substrate 1; PI3 kinase, phosphoinositol-3 kinase; AKT, protein kinase B. was significantly attenuated in IPAT in both the palm ${ }^{11}$ PrRP31 group and the palm ${ }^{11}$-PrRP31+saline group compared to saline-treated controls (Fig. 6C).

In the liver, mRNA expression of Srebf, a transcription factor of enzymes regulating de novo lipogenesis, showed a non-significant decrease in both palm ${ }^{11}$-PrRP31-treated groups compared to the saline control group (Fig. 6D), as did Acaca mRNA, which catalyzes the rate limiting step of de novo lipogenesis (Fig. 6E). Liver Lpl mRNA was significantly attenuated in the palm ${ }^{11}$-PrRP31 group and non-significantly reduced in the palm ${ }^{11}$-PrRP31+saline group (Fig. 6F). Carnitine palmitoyl transferase I (Cpt1) is a key enzyme of fatty acid oxidation in the liver. Expression of the liver-specific Cpt1a mRNA was non-significantly decreased (Fig. 6G) and that of liver-nonspecific Cpt1b mRNA was significantly lowered in both the palm ${ }^{11}$ PrRP31 group and the palm11-PrRP31+saline group compared to the saline control group (Fig. $6 \mathrm{H}$ ).

\section{Discussion}

As shown in our recent studies, lipidization of PrRP31 not only increased its stability (Maletínská et al. 2015) but also enabled PrRP31 to accomplish a central anorexigenic effect both in mice and rats with DIO (Maletínská et al. 2015, Holubova et al. 2016, Kuneš et al. 2016). In our previous studies, DIO in mice was shown to be associated with high levels of leptin (Matysková et al. 2008) and leptin resistance (Matyskova et al. 2010). In this study, the situation after discontinuation of treatment with PrRP31 was investigated because we did not find any data in the literature on anti-obesity drugs, either in current use or following withdrawal. Only Henderson and colleagues (Henderson et al. 2016) followed body weight of cynomolgus monkeys after the cessation of an 8-week treatment with a dual agonist of GLP1 and glucagon and found it to be at the pretreatment level 2 weeks after the 
The apoptotic pathway in hypothalamus - western blotting

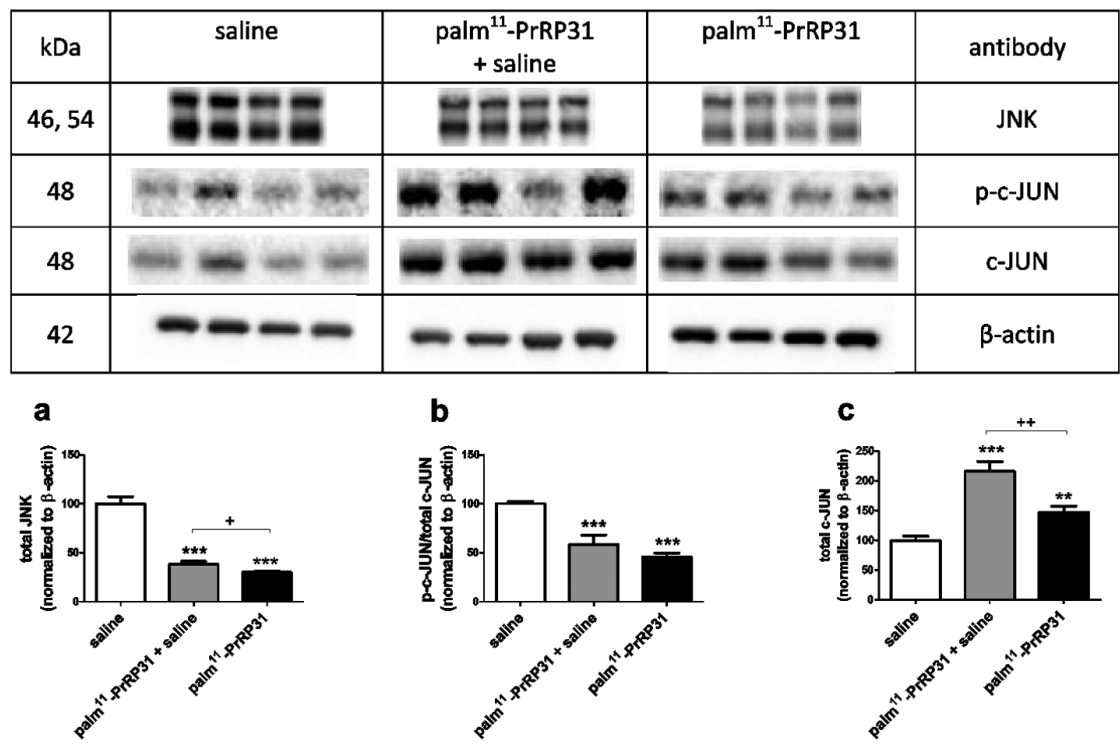

JAK2/STAT3 pathway in hypothalamus - western blotting

\begin{tabular}{|c|c|c|c|c|c|c|c|c|c|c|c|}
\hline $\mathrm{kDa}$ & \multicolumn{4}{|c|}{ saline } & \multicolumn{3}{|c|}{$\begin{array}{l}\text { palm }{ }^{11} \text {-PrRP31 } \\
+ \text { saline }\end{array}$} & \multicolumn{3}{|c|}{ palm ${ }^{11}-$ PrRP31 } & \multirow{2}{*}{$\begin{array}{c}\text { antibody } \\
\text { socs3 }\end{array}$} \\
\hline 26 & $=$ & s & $=$ & s & $=\mathbf{m}$ & $=$ & $=$ & $=0$ & $\mathbf{m}$ & $=$ & \\
\hline 79,86 & 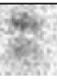 & 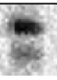 & (3) & 46 & 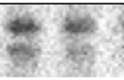 & 8 & (a) & 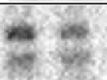 & in: & 30 & p-STAT3 \\
\hline 79 & - & 0 & $=$ & 0 & $=-$ & $\mathbf{0}$ & $=$ & -0 & $=$ & $=$ & STAT3 \\
\hline 42 & 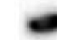 & 0 & $\infty$ & $=$ & 00 & 6 & & 50 & $\theta$ & es & $\beta$-actin \\
\hline
\end{tabular}

a

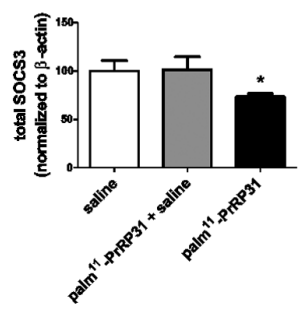

treatment. We considered a study on the palm ${ }^{11}$-PrRP31 post-treatment situation useful regarding a possible yo-yo effect after the drug termination.

In our previous studies (Maletínská et al. 2015, Holubova et al. 2016), repeated treatment of DIO rodents with palm-PrRP31 caused food intake lowering followed by gradual decrease in body weight compared to the quick drop in body weight observed after liraglutide treatment (Hansen et al. 2014). In this study, DIO mice treated for 28 days with palm ${ }^{11}$-PrRP31 (palm ${ }^{11}$-PrRP31 group) and those treated with palm ${ }^{11}$-PrRP31 for 14 days and then 14 days with saline (palm ${ }^{11}$-PrRP31 + saline group) reached a similar decrease in body weight and SCAT weight to body c

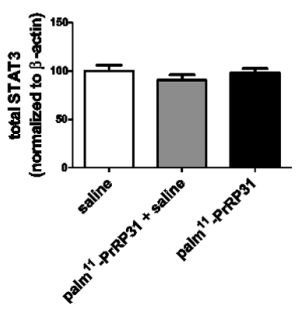

Figure 4

The apoptotic pathway in hypothalamus - total JNK, phospho-c-JUn and total c-JUN. JAK2/STAT3 pathway in hypothalamus - total SOCS3, phospho-STAT3 and total STAT3. JNK, c-JUN $\mathrm{N}$-terminal kinase. weight ratio. However, lep mRNA expression in SCAT in both palm ${ }^{11}$-PrRP31 mice and palm ${ }^{11}$-PrRP31 + saline mice did not significantly differ from that in saline control mice. On the other hand, lep mRNA expression in both palm ${ }^{11}$-PrRP31 mice and palm ${ }^{11}$-PrRP31+saline mice was significantly attenuated compared to saline control mice in IPAT whose IPAT/body weight were not changed by the treatment. It is generally accepted that plasma leptin is directly proportional to its mRNA expression (Wajchenberg 2000, Zhang et al. 2002) and that a decrease in plasma leptin results either from a lowering of particular fat depot(s), an attenuated leptin mRNA expression or both. Therefore, decreased body weight could be a consequence 
Phosphorylation of leptin receptor - western blotting

\begin{tabular}{|c|c|c|c|c|}
\hline $\mathrm{kDa}$ & saline & $\begin{array}{l}\text { palm }^{11} \text {-PrRP31 } \\
\text { + saline }\end{array}$ & palm ${ }^{11}$-PrRP31 & antibody \\
\hline 125 & $---\infty$ & $-\infty-\infty$ & $m+\cdots$ & Leptin receptor \\
\hline 100 & $=-m$ & $-m-\infty$ & $---\cdots$ & $\begin{array}{c}\mathrm{p} \text {-leptin } \\
\text { receptor } Y 1141\end{array}$ \\
\hline 42 & $-\infty-\infty$ & $-\infty-\infty$ & $-\infty-\infty$ & $\beta$-actin \\
\hline
\end{tabular}

a

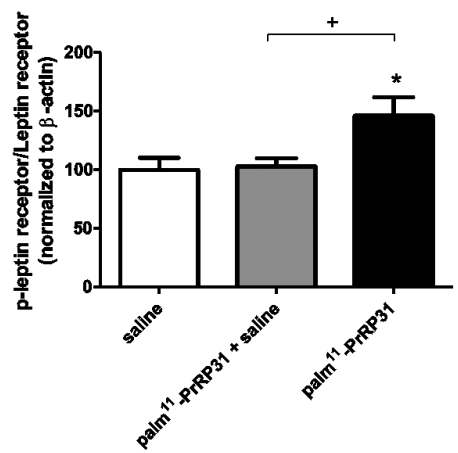

b

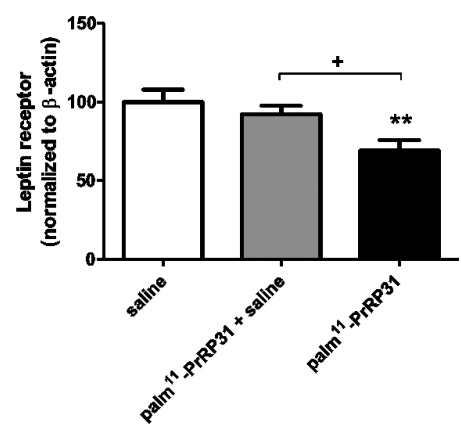

Figure 5

Phosphorylation of leptin receptor in hypothalamus. Data are presented as means \pm S.E.M. Statistical analysis was performed by unpaired $t$-test. Significance is $* P<0.05$,

$* * P<0.01, * * * P<0.001$ vs saline treated group, $+P<0.05,{ }^{++} P<0.01,{ }^{+++} P<0.001$ palm $^{11-}$ PrRP31 + saline vs palm ${ }^{11-P r R P 31}(n=8)$. of decreased SCAT and tendency of leptin mRNA decrease, despite the discontinuation of treatment. The well-known synergism between the effects of leptin and PrRP (Dodd \& Luckman 2013) could suggest possible improvement of leptin resistance by lipidized PrRP.

Changesinmetabolicparameterswere complemented with genes related to fatty acid metabolism in fat and liver. Lipogenesis in the liver could be attenuated regarding to the non-significant decrease in mRNA expression of both Acaca, the enzyme catalyzing the rate limiting step of lipogenesis and Srebf that regulates Acaca transcription. In addition, we show that mRNA expression of lipolytic enzymes $L p l$ and $C p t 1 b$ were significantly lowered by the action of palm ${ }^{11}$-PrRP31. This is in accordance with our previous study with palmPrRP31, a PrRP31 analog with palmitic acid attached to the N-terminus of the peptide that complexly attenuated liver lipid metabolism (Maletínská et al. 2015). Increased UCP1 in BAT points to the increased energy expenditure and has already been described in our previous study (Pražienková et al. 2017).

Increased neuronal activity determined by FOSB activation, a marker of long-term neuronal potentiation (Nestler et al. 2001), in both the arcuate nucleus and NTS in the palm ${ }^{11}$-PrRP31 group in this study correlated with our previous results, where c-FOS, a marker of short-term neuronal potentiation, increased after acute administration of palm-PrRP31 (Maletínská et al. 2015). Interestingly, increased FOSB was also found in NTS in the palm ${ }^{11}$-PrRP31+saline group; we could hypothesize that a decrease in the leptin level was rather durable and increased leptin-related signaling was the driving force for maintaining body weight and protecting against the yo-yo effect.

Palm ${ }^{11}$-PrRP31 attenuated HF feeding that impacted signaling in the hypothalamus, namely, increased IRS1 and AKT through an increase in PI3 kinase and enhanced ERK phosphorylation. Leptin receptor-induced PI3 kinase pathway is more sensitive to be lowered by DIO than STAT3 (Balland \& Cowley 2015). Also, in our study, STAT3 signaling was not significantly changed and SOCS3 significantly decreased after the peptide treatment. It seems that the PI3 kinase pathway was restored by the body weight-lowering effect of palm ${ }^{11}$-PrRP31. Increased ERK1/2 phosphorylation was a result of either leptin receptor or PrRP receptor signaling. Phoshorylation of leptin receptor in hypothalamus was increased only after palm ${ }^{11-P r R P 31}$ treatment.

JNK is generally activated by HF feeding in DIO mice, both in the periphery and the brain, namely, in the hypothalamus (De Souza et al. 2005). The precise mechanism of this JNK effect is still vague. Food intake and body weight in DIO mice were shown to be attenuated by a pan-JNK inhibitor administered both peripherally and intracerebroventricularly (Gao et al. 2017), and neuron-specific JNK inactivation increased hypothalamic insulin sensitivity and peripheral tolerance to glucose and insulin (Belgardt et al. 2010). In this study, palm¹1-PrRP31 attenuated DIO and in the hypothalamus lowered both JNK and c-JUN phosphorylation related to c-JUN that is 

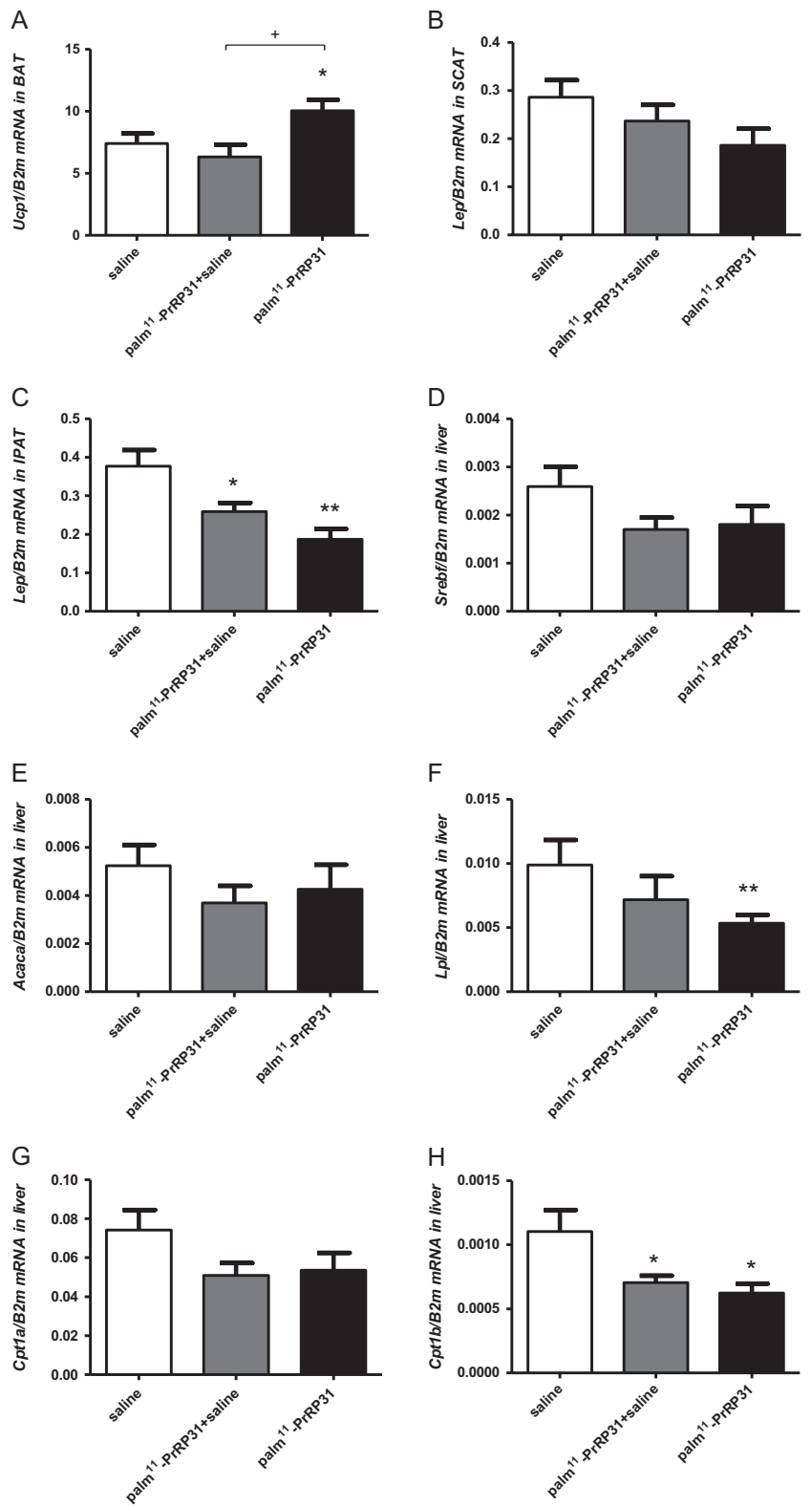

\section{Figure 6}

Chronic effect of palm ${ }^{11-P r R P 31}$ on mRNA expressions in DIO mice. (A) Ucp1 in BAT, (B) Lep in SCAT, (C) Lep in IPAT, (D) Srebf, (E) Acaca, (F) $L p l,(G) C p t 1 a$ and (H) Cpt1b in liver. Mice were subcutaneously injected twice a day with saline for 28 days (saline group), with palm ${ }^{11}$-PrRP31 $(5 \mathrm{mg} / \mathrm{kg})$ for 28 days (palm ${ }^{11-P r R P 31}$ group) or with palm 11 -PrRP31 $(5 \mathrm{mg} / \mathrm{kg})$ for 14 days and for the following 14 days with saline (palm ${ }^{11-P r R P 31}+$ saline group). After the treatment the mRNA expressions were determined. Data are presented as mean \pm S.E.M. The data were normalized to beta-2-microglobulin and analyzed by unpaired $t$-test, significance is $* P<0.05, * * P<0.01$ vs the saline treated group, $+P<0.05$ palm ${ }^{11}$-PrRP31 + saline vs palm ${ }^{11}$-PrRP31 $(n=10)$. Ucp1, uncoupling protein-1; Lep, leptin; Lpl, lipoprotein lipase; Cpt1b, carnitine palmitoyltransferase $1 \mathrm{~b}$; $B A T$, brown adipose tissue; IPAT, intraperitoneal adipose tissue; SCAT, subcutaneous adipose tissue directly regulated by JNK. This connects palm ${ }^{11}$-PrRP31's body weight-lowering effect to the hypothalamic JNK/cJUN signaling.

In conclusion, palm ${ }^{11}$-PrRP31 induced a significant decrease in body weight, SCAT weight and IPAT Lep mRNA expression resulting in an attenuated plasma leptin that continued even 14 days after termination of its 14-day-long administration and maintained increased neuronal activity in food intake-regulating neurons of the NTS and in the hypothalamus, and activated PI3 kinase and ERK1/2 pathways but deactivated the apoptotic JNK/c-JUN pathway. We conclude that normalized leptin levels retained the decrease in body weight even after the termination of treatment and prevented the yo-yo effect. Moreover, leptin-related signaling in the hypothalamus was ameliorated, and apoptotic signaling pathways were attenuated by treatment with palm ${ }^{11}$-PrRP31. Thus, palm ${ }^{11}$-PrRP31 is highly promising as a possible obesity treatment when a long-term effect is needed.

\section{Declaration of interest}

The authors declare that there is no conflict of interest that could be perceived as prejudicing the impartiality of the research reported.

\section{Funding}

This work was supported by GA CR grant No. 16-00918S, RVO:61388963 of AS CR and RVO:67985823 of AS CR. The authors would like to thank H Vysušilová for excellent technical assistance, Z Lacinová for mRNA analyses and M Blechová for synthesis of PrRP analog.

\section{References}

Arch JR 2015 Horizons in the pharmacotherapy of obesity. Current Obesity Reports 4 451-459. (https://doi.org/10.1007/s13679-0150177-4)

Balland E \& Cowley MA 2015 New insights in leptin resistance mechanisms in mice. Frontiers in Neuroendocrinology 39 59-65. (https://doi.org/10.1016/j.yfrne.2015.09.004)

Belgardt BF, Mauer J, Wunderlich FT, Ernst MB, Pal M, Spohn G, Brönneke HS, Brodesser S, Hampel B, Schauss AC, et al. 2010 Hypothalamic and pituitary c-Jun N-terminal kinase 1 signaling coordinately regulates glucose metabolism. PNAS 107 6028-6033. (https://doi.org/10.1073/pnas.1001796107)

Bray GA \& Ryan DH 2014 Update on obesity pharmacotherapy. Annals of the New York Academy of Sciences 1311 1-13. (https://doi. org/10.1111/nyas.12328)

Bray GA, Frühbeck G, Ryan DH \& Wilding JP 2016 Management of obesity. Lancet 387 1947-1956. (https://doi.org/10.1016/S01406736(16)00271-3)

De Souza CT, Araujo EP, Bordin S, Ashimine R, Zollner RL, Boschero AC, Saad MJ \& Velloso LA 2005 Consumption of a fat-rich diet activates 
a proinflammatory response and induces insulin resistance in the hypothalamus. Endocrinology 146 4192-4199. (https://doi. org/10.1210/en.2004-1520)

Dodd GT \& Luckman SM 2013 Physiological roles of GPR10 and PrRP signaling. Frontiers in Endocrinology 4 20. (https://doi.org/10.3389/ fendo.2013.00020)

Ellacott K, Lawrence C, Rothwell N \& Luckman S 2002 PRL-releasing peptide interacts with leptin to reduce food intake and body weight Endocrinology 143 368-374. (https://doi.org/10.1210/ endo.143.2.8608)

Fosgerau K, Raun K, Nilsson C, Dahl K \& Wulff BS 2014 Novel $\alpha$-MSH analog causes weight loss in obese rats and minipigs and improves insulin sensitivity. Journal of Endocrinology 220 97-107. (https://doi. org/10.1530/JOE-13-0284)

Franklin K \& Paxinos G 1997 The Mouse Brain in Stereotaxic Coordinates. New York, USA: Academic Press.

Gao S, Howard S \& LoGrasso PV 2017 Pharmacological inhibition of c-Jun N-terminal kinase reduces food intake and sensitizes leptin's anorectic signaling actions. Scientific Reports 7 41795. (https://doi. org/10.1038/srep41795)

Hansen HH, Hansen G, Paulsen S, Vrang N, Mark M, Jelsing J \& Klein T 2014 The DPP-IV inhibitor linagliptin and GLP-1 induce synergistic effects on body weight loss and appetite suppression in the dietinduced obese rat. European Journal of Pharmacology 741 254-263. (https://doi.org/10.1016/j.ejphar.2014.08.010)

Henderson SJ, Konkar A, Hornigold DC, Trevaskis JL, Jackson R, Fritsch Fredin M, Jansson-Löfmark R, Naylor J, Rossi A, Bednarek MA, et al. 2016 Robust anti-obesity and metabolic effects of a dual GLP-1/ glucagon receptor peptide agonist in rodents and non-human primates. Diabetes, Obesity and Metabolism 18 1176-1190. (https://doi.org/10.1111/dom.12735)

Holubova M, Zemenová J, Mikulášková B, Panajotova V, Stohr J, Haluzik M, Kunes J, Zelezna B \& Maletinska L 2016 Palmitoylated PrRP analog decreases body weight in rats with DIO but not in ZDF rats. Journal of Endocrinology. 229 85-96. (https://doi.org/10.1530/ JOE-15-0519)

Kuneš J, Pražienková V, Popelová A, Mikulášková B, Zemenová J \& Maletínská L 2016 Prolactin-releasing peptide: a new tool for obesity treatment 2016. Journal of Endocrinology 230 R51-R58. (https://doi. org/10.1530/JOE-16-0046)

Lawrence C, Ellacott K \& Luckman S 2002 PRL-releasing peptide reduces food intake and may mediate satiety signaling. Endocrinology 143 360-367. (https://doi.org/10.1210/endo.143.2.8609)

Maletínská L, Nagelová V, Tichá $\mathrm{A}$, Zemenová J, Pirník Z, Holubová M, Špolcová A, Mikulášková B, Blechová M, Sýkora D, et al. 2015 Novel lipidized analogs of prolactin-releasing peptide have prolonged halflives and exert anti-obesity effects after peripheral administration. International Journal of Obesity 39 986-993.

Manning S, Pucci A \& Finer N 2014 Pharmacotherapy for obesity: novel agents and paradigms. Therapeutic Advances in Chronic Disease 5 135-148. (https://doi.org/10.1177/2040622314522848)

Matysková R, Maletínská L, Maixnerová J, Pirník Z, Kiss A \& Zelezná B 2008 Comparison of the obesity phenotypes related to monosodium glutamate effect on arcuate nucleus and/or the high fat diet feeding in C57BL/6 and NMRI mice. Physiological Research 57 727-734. (https://doi.org/10.1038/ijo.2015.28)

Matyskova R, Zelezna B, Maixnerova J, Koutova D, Haluzik M \& Maletinska L 2010 Estradiol supplementation helps overcome central leptin resistance of ovariectomized mice on a high fat diet. Hormone and Metabolic Research 42 182-186. (https://doi. org/10.1055/s-0029-1243250)

Nestler EJ, Barrot M \& Self DW 2001 DeltaFosB: a sustained molecular switch for addiction. PNAS 98 11042-11046. (https://doi. org/10.1073/pnas.191352698)

Patel D 2015 Pharmacotherapy for the management of obesity. Metabolism 64 1376-1385. (https://doi.org/10.1016/j.metabol.2015.08.001)

Pirnik Z, Zelezna B, Kiss A \& Maletinska L 2015 Peripheral administration of palmitoylated prolactin-releasing peptide induces Fos expression in hypothalamic neurons involved in energy homeostasis in NMRI male mice. Brain Research 1625 151-158. (https://doi.org/10.1016/j.brainres.2015.08.042)

Prazienkova V, Ticha A, Blechova M, Spolcova A, Zelezna B \& Maletinska L 2016 Pharmacological characterization of lipidized analogs of prolactin-releasing peptide with a modified C-terminal aromatic ring. Journal of Physiology and Pharmacology 67 121-128.

Pražienková V, Holubová $\mathrm{M}$, Pelantová $\mathrm{H}$, Bugáňová $\mathrm{M}$, Pirník Z, Mikulášková B, Popelová A, Blechová M, Haluzík M, Železná B, et al. 2017 Impact of novel palmitoylated prolactin-releasing peptide analogs on metabolic changes in mice with diet-induced obesity. PLoS ONE 12 e0183449. (https://doi.org/10.1371/journal. pone.0183449)

Rodgers RJ, Tschöp MH \& Wilding JP 2012 Anti-obesity drugs: past, present and future. Disease Models and Mechanisms 5 621-626. (https://doi.org/10.1242/dmm.009621)

Roland B, Sutton S, Wilson S, Luo L, Pyati J, Huvar R, Erlander M \& Lovenberg T 1999 Anatomical distribution of prolactin-releasing peptide and its receptor suggests additional functions in the central nervous system and periphery. Endocrinology 140 5736-5745. (https://doi.org/10.1210/endo.140.12.7211)

Špolcová A, Mikulášková B, Holubová M, Nagelová V, Pirnik Z, Zemenová J, Haluzík M, Železná B, Galas MC \& Maletínská L 2015 Anorexigenic lipopeptides ameliorate central insulin signaling and attenuate tau phosphorylation in hippocampi of mice with monosodium glutamate-induced obesity. Journal of Alzheimer's Disease 45 823-835. (https://doi.org/10.3233/JAD-143150)

Takayanagi Y, Matsumoto H, Nakata M, Mera T, Fukusumi S, Hinuma S, Ueta Y, Yada T, Leng G \& Onaka T 2008 Endogenous prolactin-releasing peptide regulates food intake in rodents. Journal of Clinical Investigation 118 4014-4024. (https://doi.org/10.1172/ JCI34682)

Wajchenberg BL 2000 Subcutaneous and visceral adipose tissue: their relation to the metabolic syndrome. Endocrine Reviews 21 697-738. (https://doi.org/10.1210/edrv.21.6.0415)

Zhang Y, Guo KY, Diaz PA, Heo M \& Leibel RL 2002 Determinants of leptin gene expression in fat depots of lean mice. American Journal of Physiology: Regulatory, Integrative and Comparative Physiology 282 R226-R234. (https://doi.org/10.1152/ ajpregu.00392.2001)

Received in final form 21 November 2017

Accepted 11 December 2017

Accepted Preprint published online 12 December 2017 (c) 2018 Society for Endocrinology Published by Bioscientifica Ltd. Printed in Great Britain 